\title{
ENVEJECIMIENTO Y DISCAPACIDAD. UNA APROXIMACION AL CASO ESPAÑOL DESDE LA PERSPECTIVA DEL BIENESTAR SOCIAL
}

\author{
ESTER BÓDALO LOZANO
}

Universidad de Murcia.

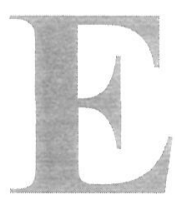

\section{INTRODUCCIÓN}

1 progresivo aumento del envejecimiento de la población en la mayoría de los países occidentales se produce fundamentalmente por el alargamiento de la esperanza de vida y por una disminución de la fecundidad. España no es ajena a este fenómeno.

Una de las consecuencias del envejecimiento demográfico es la mayor longevidad de los sujetos. Este hecho -sin duda positivo- a partir de ciertas edades lleva a que el número de personas mayores que padece algún tipo de discapacidad se haya visto incrementado notablemente en los últimos años.

El aumento de la población de personas con discapacidad que llegan a edades avanzadas es paralelo al envejecimiento demográfico de la población, derivado de los avances e innovaciones en las ciencias de la salud y en la mejora de las condiciones de vida. Como consecuencia, el envejecimiento de las personas con discapacidad presenta una serie de necesidades adicionales que van a modificar la situación anterior, añadiendo mayores complejidades.

El incremento progresivo de la esperanza de vida de las personas que padecen algún tipo de discapacidad es un fenómeno positivo en sí mismo, pero también plantea nuevos retos en los campos tanto de la investigación como de formación de políticas sociales, porque hoy en día todavía existe un desconocimiento sobre las necesidades que presentan las personas que envejecen y su entorno familiar. Pilar Rodríguez, jefa de Servicio de Estudios del IMSERSO (Rodríguez, P. 2003:33), también ve esto como un fenómeno positivo porque las personas con discapacidad van adquiriendo una mayor esperanza de vida y por lo tanto hay cada vez más personas que han vivido con una discapacidad y llegan a edades avanzadas. Este es un fenómeno que origina un reto tanto para las políticas de servicios sociales como para la investigación, 
porque todavía se conoce poco de este fenómeno que es relativamente nuevo. Antes, las personas con discapacidad grave y congénita, o bien adquirida en los primeros años de la vida, morían antes o no llegaban a la vejez.

Envejecer es un fenómeno inevitable y un proceso natural en cualquiera, pero distinto según las personas y su situación.

El ser humano alcanza la edad vital entre los veinte y los cincuenta años, a partir de ahí comienza un declive porque el número de células dañadas es mayor que las sanas. No existe el elixir de la eterna juventud porque tenemos un reloj biológico que marca nuestro ritmo de envejecimiento sincronizado a un ritmo de veinticuatro horas.

En una persona mayor sana la pérdida de masa muscular es el factor limitante que determina su posibilidad de vida independiente, y se insiste en que los últimos años hay que vivirlos en buenas condiciones, con capacidad de conocimiento y razonamiento intelectual mínimo. El envejecimiento de una persona con discapacidad es diferente porque toda su vida ha vivido en dependencia y todavía hoy existe escaso conocimiento de las necesidades que se plantean las personas con discapacidad que envejecen (Verdugo Alonso, M.A, 1995, pp .3-4).

De esta manera, caracterizamos del siguiente modo a la población mayor en España:

\section{RADIOGRAFÍA DE LAS PERSONAS MAYORES}

- Siete millones de personas tienen más de 65 años.

- Un millón y medio tiene más de 80 años.

- Alrededor de un millón de personas mayores viven solas.

- La tercera parte de los mayores necesita ayudas en las tareas cotidianas.

- En el 2001 cuatro personas activas sustentaban a un jubilado.

- La cuarta parte de la población europea superará en 2020 los 65 .

Los españoles continúan aumentando su esperanza de vida. Es por ello, por la preocupación de una calidad de vida más larga, hace que se convierta en una cuestión primordial para el bienestar del individuo, de la familia y de los responsables de las políticas sociales. Ahora lo que importa es cómo se viven estos años ganados a la muerte: la expectativa de ida con buena salud es un indicador cada vez más importante, como ha sido y lo es el de la esperanza de vida (Abellán, A.1999).

El aumento de la esperanza de vida, en paralelo al descenso de la mortalidad, es una medida incompleta o de alcance limitado para cono- 
cer la salud de una población. Algunos autores asumen que la ganancia se debe a que la gente vive mejor, es decir, con mejor estado de salud y menos discapacidad. Otros sostienen que la mayor longevidad conseguida gracias al progreso sanitario y farmacológico, trae más años vividos en fragilidad y dependencia. Se precisan más estudios que profundicen en estas dos teorías contrapuestas.

Hasta ahora las hipótesis sobre comprensión o expansión de la morbilidad y dependencia se pueden plantear de una forma más sencilla: si baja la mortalidad y aumenta la morbilidad, es presumible que exista una mayor prevalencia de situaciones de enfermedad, fragilidad y dependencia.

Envejecer con salud es un proceso que dura toda la vida y las personas con discapacidad, bien sea física o mental, necesitan formar parte de la "Sociedad Mayor", aunque en este proceso de ir envejeciendo perciban una doble experiencia, por un lado envejecen como cualquier persona, pero su propio proceso aporta ya una discapacidad.

\section{ANTECEDENTES DEL TEMA}

La preocupación por el envejecimiento de las personas discapacitadas es muy reciente. Ha sido en la década de los ochenta cuando se han multiplicado, en los países más avanzados, los servicios de atención a los discapacitados de mayor edad. Como hemos señalado antes la vida humana en general y de las personas con deficiencias en particular se ha alargado considerablemente como consecuencia de las condiciones médicas y de salud. El aumento de la esperanza de vida en distintos subgrupos de discapacitados, la mejora en la calidad tanto de los cuidados médicos como de la atención en residencias, junto a la progresiva desaparición de grandes instituciones -en muchos países, las grandes instituciones que tradicionalmente acogían a estas personas, han sido sustituidas por un régimen de alojamiento comunitario con acceso a centros y recursos que se ofrecen a nivel local- han hecho que en la última década haya aumentado el interés por la creación de servicios y por el desarrollo de investigaciones sobre personas de edad avanzada con discapacidad, planteándose la necesidad de proveer servicios y resolver necesidades no planificadas. La creciente incidencia de este grupo de población se refleja también en su aumento cuantitativo en los diversos centros residenciales. Dada la juventud del tema no hay todavía opciones institucionalizadas de servicios, presentándose ante nosotros un futuro abierto de posibilidades que es necesario explorar y 
experimentar para satisfacer, de manera eficaz y creativa, las necesidades de los discapacitados en esta edad.

Los servicios han sido lo primero en desarrollarse (por parte de las mismas instituciones) para dar respuesta a las necesidades de este colectivo de personas. Sin embargo, no han existido estudios prospectivos ni planificación de desarrollo de estos servicios. De igual manera, la investigación y los estudios que fundamentan y guían la programación han sido muy escasos. Hemos de referirnos a los años ochenta para encontrar la mayor parte de los trabajos específicos que abordan el tema. A partir de estos años se detecta una significativa productividad, generándose estudios y publicaciones específicas que paulativamente nos van ayudando a centrar las preocupaciones actuales y futuras. Dada la diversidad del tipo de residencias en las que se encuentran las personas de edad avanzada con discapacidad, muchos de estos estudios demográficos realizados a nivel internacional, han tenido en cuenta los contrastes que ofrecen las distintas prestaciones (Brady, Groenweg, Vbrancic y Mc Donal, 1998). Una preocupación fundamental de estos estudios lo constituye el hecho de que sabemos que muchas personas mayores con discapacidad pasan inadvertidas a los organismos asistenciales. Por consiguiente, las encuestas puramente administrativas subestiman las cifras y proporcionan datos en cierto modo distorsionados (Hogg, J., 1998). Asimismo, se está concediendo cada vez mayor atención a la naturaleza de las prestación de servicios y la calidad de vida de estas personas (Seltzer y Krauss, 1987).

Pero la investigación es escasa no solamente en el aspecto de los servicios, existe también un conocimiento escaso de cuáles son las características y necesidades concretas de esta población. Ni siquiera aparece un acuerdo en la propia definición o límite de edad a partir del cual podemos hablar de anciano discapacitado. Las investigaciones han de trascender estos niveles hasta centrarse de forma más concreta en la vida de las personas de edad avanzada con discapacidad y de sus familias. Un tema preocupante que ha dado pie a numerosas investigaciones (Grant, G. 1998) lo ha constituido la transición del anciano discapacitado del hogar a otro tipo de residencia, como consecuencia del envejecimiento de la familia de origen con la que vivía.

En los países desarrollados existe actualmente una preocupación por las personas de edad avanzada con discapacidad y, sobre todo, la necesidad de saber quiénes son y dónde están estas personas, así como el modo en que la Administración pública y las organizaciones de carácter no gubernamental pueden articular la respuesta más adecuada para cubrir sus necesidades. 


\section{LOS DISCAPACITADOS Y LAS POSIBLES FORMAS DE CON- VIVENCIA}

La toma de determinadas medidas en relación a los mayores discapacitados, tanto en el presente como en el futuro, estará en función del conocimiento de dónde, con quién, cómo y de qué viven éstos. A dichos efectos será necesario conocer su situación familiar.

Una importante proporción del colectivo que aquí nos ocupa continúa viviendo en su casa paterna, llevándose de este modo paralelamente su propio proceso de envejecimiento con el de sus cuidadores. La colocación tardía de los adultos mayores discapacitados en centros específicos supone un problema al que es difícil dar solución. Problema respaldado por el propio dilema que representa esta situación para los padres de edad avanzada. Las familias de personas con discapacidad se muestran reacias a ingresar a sus hijos en centros específicos y, de hecho, los intentos efectuados no han dado los resultados esperados (Breitenbach, 1991).

El momento en que se plantea en la familia el problema de la persona discapacitada que envejece suele coincidir con la disminución de los medios que los padres son capaces de manejar para hacer frente a esta carga. Este periodo de la vida familiar ha sido caracterizado así (Verdugo, M.A; Gutiérrez, B., 1995):

- El propio envejecimiento de los padres puede producir una degradación en la salud y una disminución de las fuerzas físicas.

- La viudedad puede tener como consecuencia la pérdida de un cónyuge que ayudaba.

- La disminución de los recursos (jubilación, pensión de viudedad) reduce las posibilidades de ayuda profesional.

La reticencia o falta de demanda manifestada por este tipo de padres puede comprenderse si hacemos referencia a una serie de aspectos personales que parecen estar desempeñando un papel clave:

- El papel padre-hijo: la persona discapacitada que envejece bajo tutela, o que ha permanecido siempre en familia, sigue siendo un "niño" en el ánimo de sus padres. No es fácil abandonar a un niño.

- El proyecto personal: los padres de la persona discapacitada que envejece son de edad madura, en su mayor parte libres ya de responsabilidades profesionales y familiares, su trabajo se ha consolidado, o se han jubilado, lo demás hijos ya han crecido y son autónomos. Normalmente los años que les quedan por vivir servirían para el descanso y para ocuparse de uno mismo. 
Pero estas familias pueden optar por dedicar su actual "tiempo libre" a un compromiso moral que es difícil, e incluso imposible renegar: las atenciones debidas a un hijo que no puede prescindir de ellas. Mientras pueda evitarse, no se interna a una persona a la que se quiere. Algunas de estas personas sentirán la tentación de aprovechar los últimos años para dedicar aún más atención a su hijo deficiente. Hacer que este "niño" los abandone pueda incluso privarles de un compañero y de una razón de existir.

- Un aspecto económico: los padres del adulto deficiente cuentan con las ayudas que se entregan a éste, como contribución a los gastos de la familia. Para estas personas que viven de una jubilación escasa, la colocación anticipada de su hijo en un centro puede significar la pérdida de su propia autonomía financiera.

- El duelo: los padres de una persona discapacitada que envejece no sólo deben plantearse el final de la vida de su hijo, sino preparase igualmente para su propia partida. La imagen tradicional de las instituciones en las que se colocan los ancianos atrae poco, tanto para un hijo como para uno mismo. La muerte de la persona retrasada no aporta necesariamente un alivio, ya que supone el abandono del otro. Frente a esta situación angustiosa, es más fácil decirse "mañana me ocuparé de esto".

Si bien con el aumento de edad aparece la necesidad de cuidados más intensivos o de ingresar en un hogar para ancianos, este paso debe darse únicamente cuando otras ayudas ambulatorias resultan insuficientes. En este punto surge la cuestión de si debe haber residencias especiales para ancianos discapacitados o si deben acomodarse en residencias normales. Parece ser que en las residencias normales no reciben suficientes cuidados y, frecuentemente, son discriminados por los otros residentes. En general, deben de preferirse residencias pequeñas a grandes instituciones, con la garantía de cuidados médicos y de actividad terapéutica, sin aislar a las personas con discapacidades profundas (deficiencia mental profunda) o con múltiples deficiencias, y un reconocimiento de la ayuda económica para las necesidades individuales.

Como puede deducirse de lo anteriormente expuesto, el alojamiento es un asunto clave para el bienestar del anciano y, con tendencias normalizadoras hasta donde sea posible, se ha de procurar que la oferta sea realista, que comprenda las variadas formas de vida que puede adoptar en la sociedad actual, tanto las de uso generalizado como las menos conocidas e incluso las que todavía tienen una implantación incipiente pero son deseables. La adecuada realización de este aspecto requiere conocer las preferencias de los ancianos discapacitados o sus represen- 
tantes. El contraste pertinente de estas preferencias son las necesidades objetivas, y con la disponibilidad de recursos, constituirá la base de la oferta inmediata y a medio plazo.

\section{LA ENCUESTA SOBRE DISCAPACIDADES, DEFICIENCIAS Y ESTADO DE SALUD}

En 1999 se desarrolló una nueva "Encuesta sobre discapacidades, deficiencias y estado de salud", bajo la dirección del INE, que supone una importante actualización sobre la que se llevó a cabo en 1986. En 2001 aparecieron las primeras tabulaciones importantes.

Sin embargo, ya está en vigor la segunda clasificación de discapacidades. Ésta introduce cambios en conceptos, clasificación y en la misma denominación, que pasa a llamarse "Clasificación Internacional del Funcionamiento y la Discapacidad". La nueva clasificación pone el acento en lo positivo de las situaciones. Habla de funcionamiento en vez de discapacidad, y de participación en vez de minusvalía, además de prestar atención a los factores contextuales (ambientales y personales) asociados al estado de salud, y que explican mejor las dimensiones del funcionamiento; cuerpo, individuo y social. A nivel de cuerpo los aspectos positivos son la integridad funcional y las partes del cuerpo; el aspecto negativo es la deficiencia. A nivel de individuo, los aspectos positivos son las actividades que realiza; los aspectos negativos son las limitaciones a estas actividades. A nivel social los aspectos positivos son la participación en sociedad; el aspecto negativo es la restricción en la participación.

La antigua clasificación proponía un modelo más simple. La deficiencia (pérdida o anormalidad de una función o parte del cuerpo) provoca una discapacidad en la persona (restricción o ausencia de capacidad para realizar una actividad), y esto genera una situación de desventaja en el individuo, que limita o impide desempeñar un papel social, es decir, provoca una minusvalía.

\subsection{Resultados generales de la encuesta de 1999}

En España existen 2.072 .652 personas de 65 y más años con una o varias discapacidades (INE 1999). Representan el 58,7\% de todas las personas discapacitadas españolas, que suman 3.528.220 individuos.

De menos de 6 años hay 49.577 niños $(1,7 \%)$, de $6-16$ años $(2,3 \%)$, de $17-44$ años $(14,4 \%)$ y de $45-64$ años $(23,2 \%)$. 


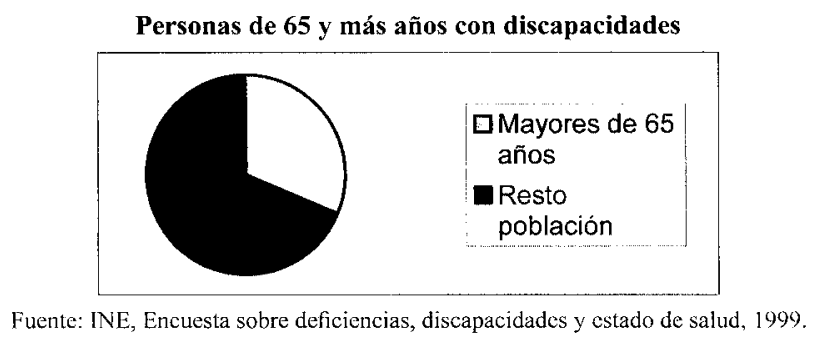

La tasa de persona de edad con discapacidad se sitúa en el $32,2 \%$, es decir, 32 personas de cada 100 mayores son discapacitados.

El patrón de la discapacidad cambia según el sexo y la edad. Hay 733.809 varones mayores que presentan discapacidad en cualquier grado y de cualquier tipo, el $27,1 \%$ de todos los varones mayores. En cambio existen 1.338.843 mujeres de edad con problemas, un $35,9 \%$ de las mujeres. La mujer mayor presenta tasas más elevadas y además entra antes en problemas y dependencia y por lo tanto en necesidad asistencial.

\subsection{Los grupos de discapacidades}

Una ojeada a la situación de los discapacitados en la encuesta de 1999, según los grupos de discapacidades, permite arrojar algo más de luz sobre las necesidades de los mismos.

Las mayores tasas de discapacidad se relacionan con la movilidad. Desplazarse fuera del hogar $(21,0 \%$ de los mayores presentan discapacidad) significa tener movilidad reducida y problemas para pasear, caminar, desplazarse en transporte público o conducir el propio vehículo. Existen 10 puntos porcentuales de diferencia entre varones y mujeres $(15,5 \%$ y $25 \%)$.

Un 15,3\% de los mayores españoles presenta incapacidades para realizar tareas del hogar (cuidar de las compras, controlar los suministros y servicios, cuidarse de las comidas, de la limpieza y cuidado de la ropa, cuidarse del bienestar de los demás miembros de la casa), sólo un $8,9 \%$ de los varones declara este problema, aunque se eleva al $20 \%$ de las mujeres. En general y referido a los mayores con discapacidad, las tareas domésticas son un problema para el $42,7 \%$ de los que se han declarado discapacitados de cualquier tipo y gravedad (la encuesta presenta un sesgo metodológico ya que los varones de estas generaciones de mayores no se han visto en la situación de tener que realizar estas tareas por lo que las cifras por sexo son tan dispares). 


\section{PERSONAS DE 65 Y MÁS AÑOS CON ALGUNA DISCAPACIDAD SEGÚN EL TIPO DE DISCAPACIDAD Y LA EDAD, 1999}

\begin{tabular}{|c|c|c|c|c|c|c|}
\hline & Total & 65-69 años & $70-74$ años & 75-79 años & 80-84 años & 85 y más \\
\hline Tareas visuales de conjunto & 361.184 & .760 & 72.838 & 90.309 & 9.597 & 73.680 \\
\hline Escuchar el habla & 581.063 & 89.068 & 112.922 & 135.691 & 108.346 & 132.036 \\
\hline Recordar inform & 291.977 & .269 & 47.005 & 61.275 & 3.918 & 87.511 \\
\hline Levantarse, acostarse & 663.635 & 115.090 & 134.155 & 141.930 & 119.411 & 153.049 \\
\hline Desplazarse dentro del & 561.491 & 74.647 & 96.564 & 121.563 & 114.307 & 154.409 \\
\hline $\begin{array}{l}\text { Utilizar utensilios, } \\
\text { herramientas }\end{array}$ & 403.500 & 62.001 & 77.285 & 82.179 & 78.791 & 103.244 \\
\hline $\begin{array}{l}\text { Desplazarse en trans } \\
\text { público }\end{array}$ & 1.182 .059 & 163.148 & 220.102 & 267.209 & 234.170 & 297.430 \\
\hline Asearse solo & 502.458 & 46.507 & 68.292 & 94.474 & 110.518 & 182.667 \\
\hline Controlar las necesidades & 250.776 & 19.093 & 30.856 & 40.098 & 57.081 & 103.648 \\
\hline Vestirse-Desvestirse & 419.754 & 44.782 & 57.139 & 75.276 & 91.502 & 151.055 \\
\hline Comer y beber & 154.536 & 9.903 & 19.226 & 26.179 & 36.069 & 63.159 \\
\hline Cuidarse de las comidas & 599.028 & 56.708 & 80.049 & 113.912 & 132.984 & 215.375 \\
\hline $\begin{array}{l}\text { Limpieza y mantenimiento } \\
\text { de la casa }\end{array}$ & 884.110 & 110.257 & 154.498 & 183.621 & 182.482 & 253.251 \\
\hline
\end{tabular}

Fuente: INE, Encuesta sobre deficiencias, discapacidades y estado de salud, 1999.

En relación a las personas de mucha edad ( 85 y más años) dos de cada tres personas tienen alguna discapacidad, no pueden realizar la limpieza y el mantenimiento de la casa. La ayuda domiciliaría es ahí donde tiene un papel fundamental para mantener a la persona cerca de su entorno el mayor tiempo posible, ya que a estas edades el porcentaje de viudas y personas viviendo en soledad es muy alto, de forma que se verían obligados a un cambio residencial o a una institucionalización.

Los problemas para levantarse, acostarse o moverse dentro del hogar afectan al 12,6\% de los mayores y, como siempre, en mayor proporción a las mujeres. Si nos referimos a los 2 millones de mayores que han confesado alguna discapacidad, el 32,0\% de ellos tiene problemas para levantarse o acostarse y moverse dentro del hogar (27-34\% varones y mujeres). Estos problemas de movilidad les hacen candidatos a no salir de casa, a renunciar a la participación social y a ponerse en riesgo de exclusión social.

Controlar las necesidades y utilizar sólo el servicio es una discapacidad que aumenta con la edad, aunque "sólo" afecta al 27,2\% de las personas de 85 y más años, les sigue en importancia las tareas de aseo, cuidarse, levantarse, etc., cuya tasa crece cuatro veces desde el 12,1\% 
de personas de 65 a 69 años que tienen discapacidad hasta el 47,9\% de 85 y más años.

\subsection{El origen de la discapacidad}

Las discapacidades entre los mayores proceden fundamentalmente de las deficiencias ostoarticulares (cuello, columna vertebral o extremidades). 757.413 mayores declaran tener discapacidad con ese origen, un $11,8 \%$ de todos los mayores, constituyéndose el problema funcional más importante. Con menor intensidad, las deficiencias visuales afectan al $8,8 \%$ de los mayores, las auditivas al 8,4\%. Un 6,1\% de las discapacidades proceden de las deficiencias mentales y al sistema nervioso, aunque los porcentajes están lejos de los problemas osteoarticulares, las consecuencias de estas discapacidades son muy graves.

\section{DEFICIENCIAS EN PERSONAS DE 65 Y MÁS AÑOS SEGÚN LAS PRINCIPALES CAUSAS QUE LAS ORIGINARON}

\begin{tabular}{|l|c|c|c|c|c|c|}
\hline & Total & Perinatal & Accidente & Enfermedad & Otras causas & No consta \\
\hline TOTAL & 3.151 .458 & 65.091 & 220.894 & 2.144 .474 & 671.392 & 49.606 \\
\hline Def mentales & 228.396 & 6.478 & 4.354 & 143.543 & 40.042 & 33.978 \\
\hline Def. visuales & 569.577 & 18.288 & 33.371 & 417.818 & 95.762 & 4.338 \\
\hline Def. del oído & 546.810 & 21.070 & 25.975 & 356.372 & 136.352 & 7.041 \\
\hline $\begin{array}{l}\text { Def del lenguaje, } \\
\text { habla y voz }\end{array}$ & 31.733 & 74 & 1.626 & 24.626 & 4.964 & 443 \\
\hline Def. osteoarticulares & 901.307 & 9.771 & 138.071 & 659.896 & 93.011 & 557 \\
\hline Def. sist. nervioso & 179.664 & 3.358 & 10.629 & 141.910 & 21.243 & 2.524 \\
\hline Def. viscerales & 217.370 & 4.903 & 2.886 & 190.580 & 18.780 & 222 \\
\hline Otras deficiencias & 476.601 & 1.149 & 3.982 & 209.729 & 261.238 & 503 \\
\hline
\end{tabular}

Fuente: INE, Encuesta sobre deficiencias, discapacidades y estado de salud, 1999.

Las enfermedades del sistema osteoarticular no son letales. Sin embargo, son las que causan los mayores problemas de funcionamiento, de realización de las actividades de la vida diaria y de participación en la sociedad. Son las que originan la mayor dependencia.

La causa de la deficiencia suele residir en la enfermedad: el 73,2\% de los mayores con deficiencias osteoarticulares sufren estas dolencias como consecuencia de una enfermedad, y un 15,3\% como resultado de un accidente, otras causas menos relevantes (congénitas, laborales u otros tipos).

Existen otros factores de riesgo de discapacidad y dependencia, cuanto menor es el nivel de instrucción mayor es el riesgo, así entre los 
universitarios se encuentran las tasas más bajas de dependencia (de diez sólo uno) mientras que las tasa entre analfabetos son muy elevadas (dos de cada tres analfabetos son dependientes).

El nivel de ingresos y estatus social repiten el mismo patrón. En los hogares con ingresos por debajo de $60.000 \mathrm{ptas} / \mathrm{mes}$ la incidencia de la dependencia es el doble que en los que tenían un nivel de ingresos más alto.

\subsection{Quién cuida a las personas mayores dependientes}

La respuesta a las situaciones de discapacidad y necesidades asistenciales se llaman cuidados, son el sistema de servicios provistos por el sector informal o formal para atender en casa o en una institución a las personas con necesidades funcionales y que no pueden realizar actividades cotidianas, habituales para el resto de la población.

Las mujeres dependientes son cuidadas ante todo por sus hijas $(36,7 \%)$, otros parientes $(19,7 \%)$, el propio marido (14,9\%), un hijo $(6,3 \%)$ y empleados del hogar, profesionales de empresas de servicios o los servicios sociales, éstos en muy baja proporción $(3,7 \%)$.

¿QUIÉN CUIDA A LOS MAYORES CON DISCAPACIDAD?

\begin{tabular}{|l|c|c|c|}
\hline Cuidador principal & Total & Varones & Mujeres \\
\hline Total & 1.053 .336 & 300.028 & 753.308 \\
\hline Cónyuge & 246.027 & 133.677 & 112.350 \\
\hline Hija & 340.001 & 63.866 & 276.135 \\
\hline Hijo & 61.828 & 14.120 & 47.707 \\
\hline Hermana & 25.331 & 6.365 & 18.966 \\
\hline Hermano & 3.386 & 611 & 2.775 \\
\hline Madre & 0 & 0 & 0 \\
\hline Padre & 0 & 0 & 0 \\
\hline Otro pariente & 155.720 & 29.252 & 126.468 \\
\hline Empleado & 91.608 & 16.446 & 75.162 \\
\hline Amigos y vecinos & 20.308 & 3.414 & 16.894 \\
\hline Huésped & 455 & 0 & 455 \\
\hline Servicios sociales & 33.954 & 6.234 & 27.720 \\
\hline Otra relación & 12.571 & 2.372 & 10.199 \\
\hline No consta & 62.146 & 23.671 & 38.476 \\
\hline
\end{tabular}

Fuente: INE, Encuesta sobre discapacidades, deficiencias y estado de salud 
Los varones dependientes son cuidados en primer lugar por su mujer $(44,6 \%)$, una hija $(21,3 \%)$ y otros familiares $(12 \%)$; el hijo y la posible nuera juegan un papel menor en este sistema de cuidados.

La ayuda formal alcanza al $13 \%$ de las personas con discapacidad que necesitan ayuda. Los servicios sociales $(3,2 \%)$ siguen siendo insignificantes estadísticamente hablando. La ayuda formal es el conjunto de servicios provistos por entidades y organizaciones, de naturaleza pública o privada, con o sin ánimo de lucro, por voluntarios y por personas que trabajan por su cuenta de forma remunerada, para cubrir las necesidades de las personas dependientes en casa, en la comunidad o en una institución.

Es decir, el modelo de atención a la discapacidad de los mayores se basa en la mujer, esposa o hija, cuidadora de varón, esposo o padre, y también mujer cuidadora de madre dependiente.

Cuando la cuidadora principal es la mujer, la esposa, un 50,3\% declara dedicar más de 40 horas semanales a atender. Cuando se trata de la hija los porcentajes también son altos (42,9\% dedican más de 40 horas semanales a cuidar de su padre o madre discapacitada). Los hijos varones, además de prestar ayuda en otro tipo de tareas, dedican menos tiempo; así, la mayor parte de ellos, el 41,3 dice ayudar una media de 1-2 horas diarias en contraste con las hijas cuidadoras.

En los mayores, la discapacidad sobreviene cuando ya son mayores $\mathrm{y}$ viven solos o en pareja. En el resto de la población sólo un 4,9\% de los discapacitados de 6-54 años vive sólo, generalmente se trata de personas que han arrastrado una deficiencia que da lugar a discapacidad mientras vivían en familia (problemas genéticos, accidentes de tráfico, accidente laboral), por lo que es extraño que se queden viviendo solos o en pareja. Este contraste de cifras llama la atención sobre las condiciones de vida de los mayores con discapacidad. La soledad acentúa el riesgo de aislamiento social, por lo que la discapacidad y la dependencia genera exclusión social.

Los datos expuestos reflejan la trascendencia cuantitativa del colectivo de personas mayores con discapacidad. Los cambios de patrones en la expectativa de vida, debida fundamentalmente a los avances médicos, tecnológicos, terapéuticos y rehabilitadores han posibilitado que este colectivo vaya en aumento.

Nos encontramos, pues, ante uno de los principales retos de este nuevo milenio si queremos conseguir que estas personas con discapacidad, mayores, sean miembros plenos de la sociedad en que viven, 
objeto que se alcanzará cuando, en la medida de sus posibilidades, estas personas alcancen el máximo nivel de autonomía e independencia.

\section{BIBLIOGAFIA}

ABELLÁN, A. (1999). Informe sobre la encuesta de deficiencias, discapacidades y minusvalias. Cap. 2 "Longevidad y Estado de Salud".

BRADY, D.E. :Groeneweb,G.:Vrbrancic., y Macdonald;L. (1988). "A comparision of the Service Needs of Community and Institutionaily based Order Person with handicap" informe presentado al Vill Congreso de IASSMD, Dublín, Irlanda, Agosto.

BREITENBACH, N. (1999). La colocación tardía de los adultos discapacitados que envejecen, Siglo Cero, núm 133, pp.18-20.

GRANT, G. (1988). "Leting Go: Tracing Reasons of the different Attitudes of Informal Carers towads the Future Care of People Handicap, informe presentado al VIII Congreso de IASSMD, Dublín, Irlanda, Agosto.

INE (1999) Encuesta sobre deficiencias, discapacidades y estado de salud.

RODRIGUEZ, P. (2003). Revista Sesenta y más, múm. 22, pp. 33.

VERDUGO ALONSO, M.A. (1995). Personas con discapacidad. Perspectivas psicopedagógicas y rehabilitadoras. Siglo Veintiuno de España Editores, S.A.

VERDUGO, M.A. y GUTIÉRREZ, B. (1995). "Retraso mental y envejecimiento". En Personas con discapacidad. Perspectivas psicopedagógicas y rehabilitadoras. Dir. Verdugo Alonso, M.A. Siglo Veintiuno de España Editores, S.A. 